\title{
Development of a Biofidelic 'Legform' Impact Test Device
}

\author{
John Davis and Peter Schuster \\ Califomia Polytechnic State University, San Luis Obispo
}

Copyright (0) 2007 SAE International

\begin{abstract}
Current EuroNCAP test specifications attempt to predict pedestrian lower limb injury in a lateral impact with a rigid legform test device developed by the UK's TRL (Transportation Research Lab). Research shows that the measurements taken from this device (knee bending angle, knee shear, and upper tibia acceleration) do not necessarily correspond to accurate injury prediction. Recent research suggests that the primary improvement to the current test device would be a flexible legform, or one that has more biofidelity (i.e., simulates actual human lower limb response).
\end{abstract}

The work presented in this paper first reviews current legforms developed for pedestrian impact testing, including the TRL impactor used in EuroNCAP tests, Honda's POLAR II pedestrian dummy, and JAMA/JARI's FLEX-PLI legform impactor. Component level testing shows the FLEX-PLI performance to be close to the human lower limb response corridors. However, there are still areas of potential improvement with this design. To address these areas, this research includes the phase 1 development of a new legform impactor incorporating adjustable ligament pre-load, direct ligament strain measurements, adjustability in knee flexion to account for the gait cycle, tuned composite bone cores that match the force-deflection curves of PMHS testing, and a condyle load plate that measures knee joint compressive forces. Development techniques include solid modeling, computer-aided manufacturing, composite design and analysis, sensor specifications, and data acquisition. In so doing, the injury specifications set by the EEVC will be modified to account for the more accurate injury assessment of the improved device.

\section{INTRODUCTION}

According to the NHTSA, 4800 pedestrians were killed and 71000 were injured in the US in 2002 . About $80 \%$ of pedestrian accidents were at speeds below $35 \mathrm{mph}$ [1]. In the European Union, 8718 pedestrians were killed and 176385 injured [2]. Using databases from countries such as Australia, Germany, Japan, and the United States over 9300 pedestrian-vehicle accident injuries were studied by the IHRA (International Harmonized
Research Association) [3]. The results from this study show that the head and lower limbs comprised $84 \%$ of the total injuries. Of the lower limb injuries, $42 \%$ were due to tibia fractures, $18 \%$ to knee injuries, and only $2 \%$ to femur fractures. The study found a strong correlation between frequency and type of injuries and vehicle frontend design. Femur and pelvis injuries from newer vehicles have been reduced dramatically over the last decade [4] due to increasingly sloped front ends.

Automobile manufacturers and independent research organizations have carried out pedestrian impact testing since the early 1980's when the EEVC's WG7 (European Enhanced Vehicle-Safety Commission Working Group 7) developed a pedestrian impact dummy to be used in crash tests. This crash test dummy was developed based on results from the EEVC's WG1, which had identified the usefulness of a pedestrian crash test [5]. Over the next decade the EEVC continued to improve on testing procedures in conjunction with various other automotive and research groups such as ACEA (European Car Manufacturer's Association), TRL (Transportation Research Lab), EuroNCAP (European New Car Assessment Program), ERGA (European Regulations Global Approach), IHRA (International Harmonized Research Association), and others [3,6]. In 1987, the EEVC established WG10 (Working Group 10) to improve on existing pedestrian crash test procedures. This group formulated three sub system tests to simulate pedestrian impact: legform to bumper, upper legform to bonnet, and headform impactor to bonnet. These tests are designed to assess the effectiveness of vehicle front ends as they relate to lower limb, pelvis, and head impact injuries.

The legform impactor that is currently used for legform to bumper tests was developed by TRL. It consists of upper and lower rigid metal cylinders-representing the thigh and lower leg-connected by deformable knee shear and bend element. The stiffness properties of the knee element were chosen to simulate PMHS (Post Mortem Human Subject) test data. This legform impacts a vehicle front end at $40 \mathrm{~km} / \mathrm{hr}$ in an effort to simulate a lateral pedestrian collision. Physical characteristics such as total mass, centers of mass for the upper and lower elements, and length segments are based on anthropometric data of the $50^{\text {th }}$ percentile male. Measurements taken from this legform include knee 
bending angle, knee shear, and tibia acceleration. Injury tolerances from these measurements have been defined by the EEVC and consist of:

- Knee bending angle $<15^{\circ}$

- Knee shear displacement $<6 \mathrm{~mm}$

- Upper tibia acceleration < $150 \mathrm{~g}$

There are many issues concerning the accuracy of this legform in predicting pedestrian injury. Component level testing conducted by UVA (University of Virginia) of the knee element and upper and lower leg segments shows that this legform does not accurately simulate human lower limb response [7]. The aim of the research described in this paper is to improve upon this device by developing a new legform impactor that has a more biofidelic response to the pedestrian impact environment.

\section{PMHS TEST RESULTS}

The EEVC injury acceptance levels were developed with a combination of PMHS testing and accident reconstruction [8]. These criteria are largely based on whole limb testing carried out by Kajzer et al. [9], who simulated a pedestrian lateral impact in a series of low and high speed $(40 \mathrm{~km} / \mathrm{hr})$ sled tests. The high speed test results are particularly relevant since the EEVC legform test is conducted at an impact velocity of 40 $\mathrm{km} / \mathrm{hr}$. A total of ten tests were performed on cadaver right lower limbs with an average age of 51 years (nine males, one female). The lower limb was subjected to a preload of $400 \mathrm{~N}$. Shearing test results showed a high risk of articular fracture of the proximal tibia and the femoral condyles as well as ACL ligament avulsion with initial damage occurring at $16 \mathrm{~mm}$ (SD 7) of shearing displacement resulting in $2.6 \mathrm{kN}$ (SD 0.5) of shear force and $489 \mathrm{Nm}$ (SD 141) of knee bending moment. Bending tests resulted in two primary injury mechanisms: Femur fractures occurred in $70 \%$ of the cases with a mean maximum shearing force of $1.4 \mathrm{kN}$ (SD 0.6) and bending moment of $351 \mathrm{Nm}$ (SD 89) at a $16.4^{\circ}$ (SD 4.4) bending angle. $20 \%$ of the tests resulted in $\mathrm{MCL}$ avulsion (note: direction of impact was at the medial side) and a low frequency of $A C L$ and PCL avulsion. Initial damage in these cases occurred at a $14.6^{\circ}$ (SD 0.2) bending angle with $1.4 \mathrm{kN}$ (SD 0.2) of knee shear force and $284 \mathrm{Nm}$ (SD 18) of knee bending moment.

\section{ADDITIONAL KNEE TESTING}

In addition to the whole limb lateral impact tests performed by Kajzer et al., there have been many isolated knee joint tests performed in order to find the response of the knee joint and ligaments without additional factors affecting these tests such as impact location and thigh or lower leg inertia, whose effects could outweigh knee joint stiffness. Takahashi et al [10] states that the impact tests performed by Kajzer et al. did not show any noticeable difference in the force-time history even when the knee joint stiffness was increased by 4.5 times. The contribution of knee joint stiffness to the force-time history is small and primarily dominated by the inertial effects of the legform and the stiffness of the impacting surface.

Much of the most recent lower limb testing has been carried out at the University of Virginia. Kerrigan et al. [11] performed a series of lower limb tests to establish injury criteria for a pedestrian impact. Using five knee specimens isolated from the lower limb, the bending response from 3-point and 4-point bending could be determined without these inertial effects. 4-point bending tests resulted in a knee bending failure moment of $136.9 \mathrm{Nm}$ (SD 5.5) at $12^{\circ}$ (SD 1.1), which is much less than that reported by Kajzer et al of $284 \mathrm{Nm}$ at $14.6^{\circ}$. The initial injury mechanism was $\mathrm{MCL}$ ligament avulsion but $A C L$ tears and femoral condyle fractures were reported as well. Shear tests resulted in ACL avulsion as the primary injury mechanism occurring at $27.7 \mathrm{~mm}$ (SD 16.9) of actuator displacement with a corresponding shear force of $1115.3 \mathrm{~N}$ (SD 780.7).

In additional tests carried out by Bose et al [12], sixteen knee specimens were subjected to 3-point and 4-point bending (eight each). In order to simulate real world pedestrian accidents where the impact location varies due to bumper height and pedestrian size, the 3-point bending tests were conducted by varying the location of the supports in relation to the impactor tip. This varied the bending moment and shear force proportions resulting in different injury mechanisms. In order to replicate a $40 \mathrm{~km} / \mathrm{hr}$ pedestrian impact, the kneebending rate was determined to be $1 \% \mathrm{~ms}$. All 4-point bending tests resulted in either partial or complete avulsion of the MCL at about $130 \mathrm{Nm}$ of knee bending moment. In the varying-support-location 3-point bending tests, MCL avulsion was the primary injury mechanism with one specimen resulting in a slight $A C L$ tear.

\section{ADDITIONAL LOWER LIMB TESTING}

In addition to the knee testing performed by Kerrigan et al. [11], femur and tibia 3-point bending tests were conducted in order to establish pedestrian lower limb injury criteria. Maximum bending moment for the tibia subjected to lateral-medial 3-point loading was $310 \mathrm{Nm}$ (mean) with a mean distance between supports of 351.3 $\mathrm{mm}$. Femur bending tests showed a failure moment of $411 \mathrm{Nm}$ with a support span of $399 \mathrm{~mm}$. Nyquist et al. [13] conducted static and dynamic 3-point bending tests of the human lower leg (tibia, fibula, and surrounding flesh). The support span of these experiments was 250 $\mathrm{mm}$ and a total of 22 tibias were tested. It was found that bending strength was independent of the direction of loading (lateral-medial or anterior-posterior) and failure occurred due to tensile stresses. The lateralmedial loading curve consisted of two slopes with the first being due to the flesh and fibula surrounding the tibia. The slopes for these two curves are $105 \mathrm{~N} / \mathrm{mm}$ and $265 \mathrm{~N} / \mathrm{mm}$, respectively. Mean failure bending moments were found to be $320 \mathrm{Nm}$ for males and 280 
$\mathrm{Nm}$ for females. These values compare favorably to those found by Kerrigan et al. of $310 \mathrm{Nm}$.

\section{CURRENT TEST DEVICES}

In an effort to gain an understanding of how legforms predict a pedestrian injury, an overview of the structure and injury prediction methodology for several current legforms is presented in the following section.

\section{TRL RIGID LEGFORM}

The TRL rigid legform impactor shown in Figure 1 is the current pedestrian impact test device that the EEVC uses to test the effectiveness of a vehicle front end in reducing pedestrian injury. This device consists of rigid upper and lower segments and a deformable shear spring that is meant to simulate the response of the human knee in a lateral impact. Injury assessment consists of measuring the deformation angle and shearing displacement between the upper and lower segments $\left(<15^{\circ}\right.$ and $6 \mathrm{~mm}$, respectively) in addition to the acceleration of the upper tibia $(<150 \mathrm{~g}) 66 \mathrm{~mm}$ below the knee joint. The assembly is surrounded by $25 \mathrm{~mm}$ of Confor foam that is meant to simulate human lower limb soft tissue. Physical properties are designed to correspond to those of the $50^{\text {th }}$ percentile male.

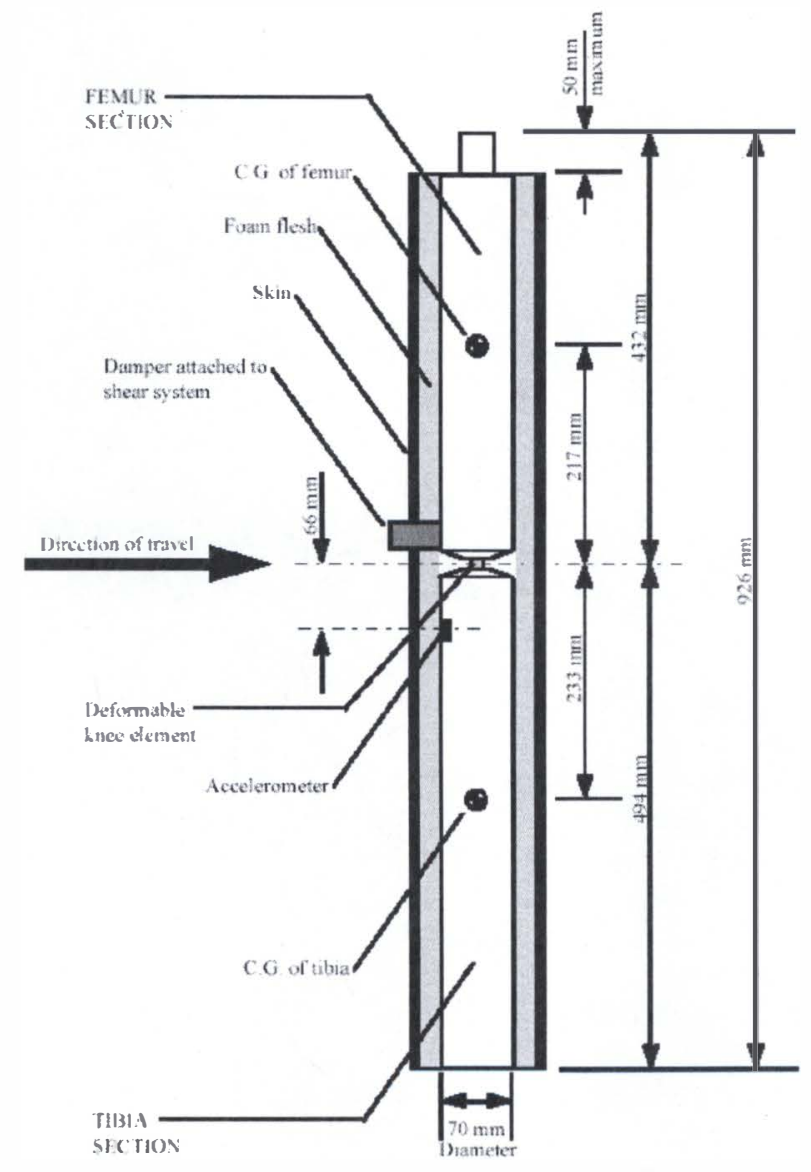

Figure 1. TRL rigid legform impactor [8]
In a series of knee joint tests conducted by Bhalla et al. [7], the bending and loading response of the TRL impactor was compared to that of PMHS knee joints. In pure 4-point bending tests, human knee joint tests showed failure starting at an average of $12.7^{\circ}$ bending angle and $143 \mathrm{Nm}$ of bending moment. At this bending angle, the TRL legform measured over $470 \mathrm{Nm}$, more than three times the stiffness of human knee joints. The maximum knee shear displacement capability of the TRL is $8 \mathrm{~mm}$ (EEVC injury prediction occurs at $6 \mathrm{~mm}$ ) but comparable human knee joints showed a shearing displacement of $12.7 \mathrm{~mm}$ minimum at first injury.

Schuster [14] developed a finite element model of both the human lower limb and the TRL legform impactor. Pedestrian impact simulations between the two models were conducted and analyzed. Recommendations were made to improve the TRL impactor, including: Reducing knee joint stiffness, incorporating a flexible tibia member with strain gauges, and increasing the energy absorbing capabilities of Confor foam. The $25 \mathrm{~mm}$ thick foam that covers the legform is of constant thickness. It is recommended to decrease this thickness at the knee joint area to better simulate the soft tissue properties at this location, and increase it elsewhere in the legform. In addition to these proposed changes to the legform itself, Schuster also recommended modifying the EEVC injury criteria by increasing the maximum knee shear above $6 \mathrm{~mm}$ and decreasing the maximum knee bend angle below $15^{\circ}$.

Konosu et al. [15] studied the TRL legform impactor and EEVC injury criteria and concluded that a flexible legform was needed for correct impact response. They also noted that the maximum tibia acceleration criterion of $150 \mathrm{~g}$ was obtained using a constant impact height. If the bumper to legform impact location is changed, injuries could still result with lower or higher accelerations measured by the fixed-height legform accelerometer.

\section{POLAR II PEDESTRIAN DUMMY}

Honda R\&D initially developed the POLAR pedestrian dummy by combining features from existing in-car dummies, such as THOR, to create a full-scale pedestrian test device (see Figure 2). The thigh segment is composed of a rigid element that is mounted with load cells to measure axial loads and accelerometers (linear and angular) to measure displacements and bending angle. The lower leg consists of a flexible tibia member that is constructed from a nylon/Kevlar core with a urethane outer rod that is meant to correspond to the stiffness of the human lower limb. The knee element consists of femoral condyles that mate to a meniscus mounted on the tibial plateau. Coil springs with cables are used to simulate the ligaments $[10,16]$.

Bhalla et al. [7] performed the same series of tests (knee bending and shear) on the POLAR II as on the TRL rigid. Bending test results show that the POLAR knee 
joint is still stiffer than that of PMHS knee joints but lower than the TRL. Shear tests resulted in higher shearing stiffness than PMHS results but the shear displacement of the POLAR is sufficient and not limited by maximum travel. Takahashi et al. [10] performed component level testing of the POLAR knee joint and flexible tibia and compared this to FEA simulations. The slope of the tibia force-deflection curve in 3-point bending was estimated to be $215 \mathrm{~N} / \mathrm{mm}$. This stiffness is less than that shown in PMHS tibia tests [13], which showed a tibia bending stiffness of $265 \mathrm{~N} / \mathrm{mm}$, but still shows good biofidelity especially when compared to the TRL rigid lower leg.

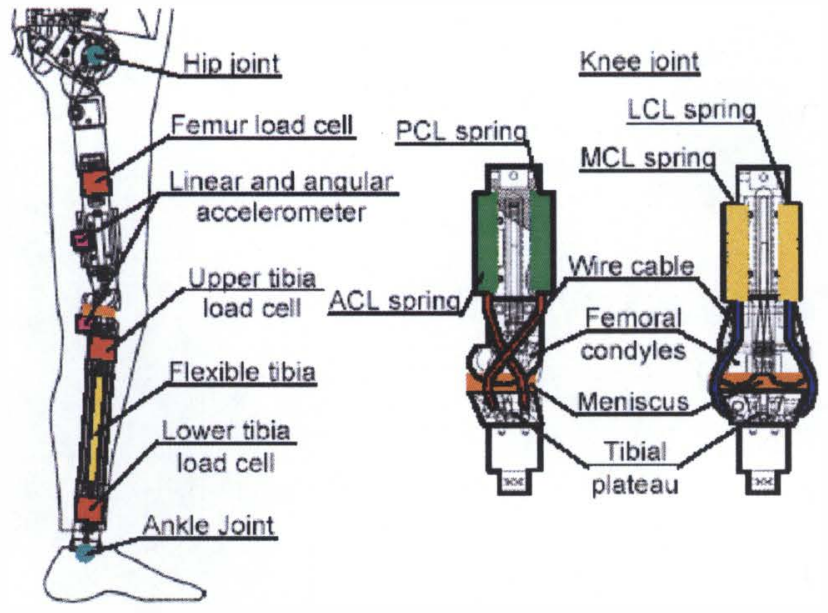

Figure 2. POLAR II lower limb details showing the knee element as well as the upper and lower components [2].

When compared to the TRL rigid legform, the POLAR II shows great improvement with flexibility of the lower leg, increased shear displacement, and lower knee bending stiffness. In terms of injury prediction, some areas of concern are the ability to accurately measure kneebending angle from the angular accelerometers and knee shear from the linear accelerometers.

\section{JAMAJARI FLEX PLI 2003}

The FLEX PLI 2003 shown in Figure 3 is a third generation design that features greater injury prediction capabilities than any of the other previous legforms. The physical properties match those of the $50^{\text {th }}$ percentile male and both the thigh and lower leg are composed of flexible elements. Konosu and Tanahashi [17] detail the development of the FLEX PLI that includes component level testing of the thigh, lower leg, and knee.

The structure of the knee joint consists of compressive springs (76 N/mm lateral/medial and $164 \mathrm{~N} / \mathrm{mm}$ anterior/posterior) and cables simulating the cruciate ligaments with potentiometers that measure ligament deflection. A tibial plateau rests on two load cells that measure compressive forces in the knee joint. Component level testing shows good correlation between the knee joint bending stiffness and PMHS results [11], although it is recommended to include pre- tensioning of the ligament springs to increase the biofidelity of the knee joint bending response [18].

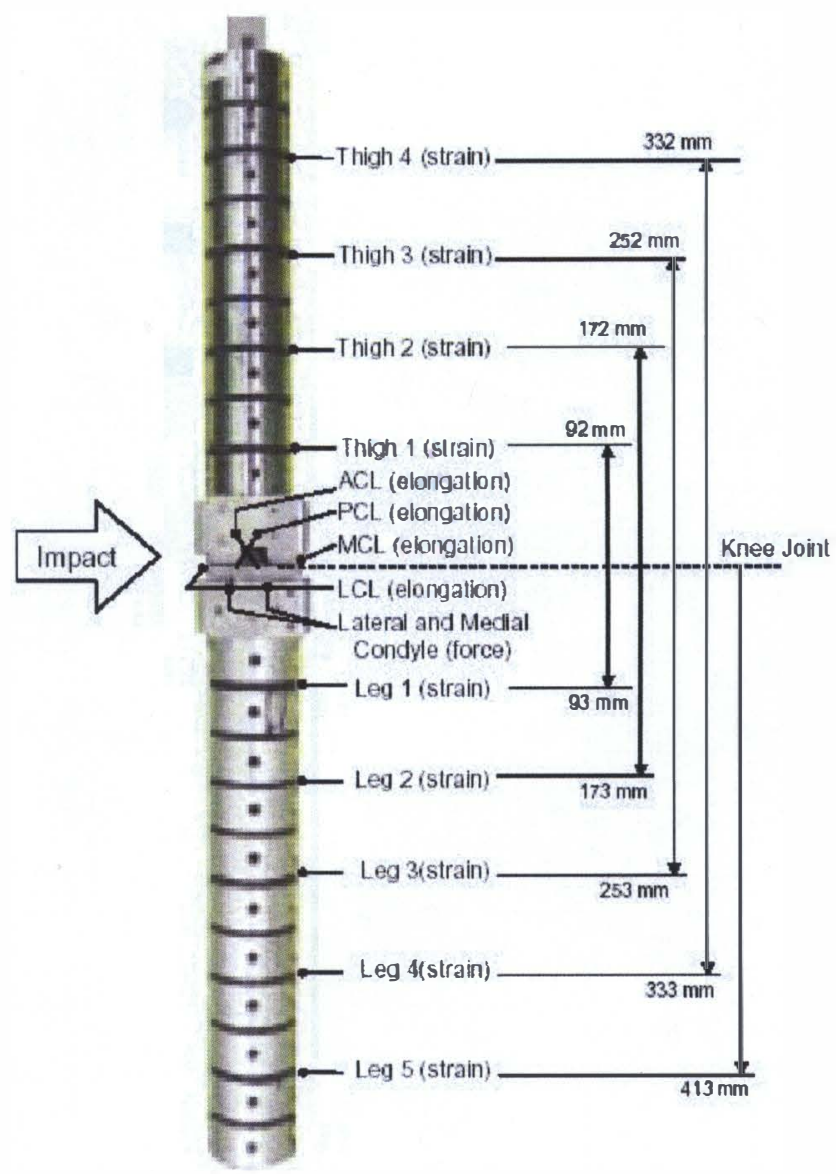

Figure 3. FLEX PLI 2003 incorporates flexible thigh and lower leg with deformable knee joint [9].

The upper and lower segments consist of fiber reinforced bone cores that are surrounded by urethane strips on the lateral-medial sides and held together by binders. Aluminum collars allowing lateral-medial flexibility are fitted over both segments. Component level testing of the fiber reinforced bone cores and the whole lower limb assembly shows good correlation when compared with the PMHS lower limb tests performed by Kerrigan et al. [11]. Injury sensing capabilities of the thigh and lower leg include multiple strain gauges mounted on the fiber reinforced bone cores and accelerometers mounted in the aluminum segments.

\section{DESIGN AND ANALYSIS}

Legform impactors such as the POLAR II and FLEX PLI have been developed in an effort to increase the biofidelity of a pedestrian test device. While these devices exhibit a biofidelic response to lateral impacts that occur in a pedestrian environment, the injury prediction capabilities and tolerances have been identified as areas that can be improved upon. For instance, the POLAR II legform does not have any means of predicting upper or lower bone bending 
strains. The FLEX PLI incorporates strain gauges in the bone cores of the femur and tibia but is only capable of measuring deformations in the lateral-medial loading direction. No mechanism exists in the POLAR II knee joint for directly measuring ligament strains or intercondyle compressive forces. The FLEX PLI addresses these issues by incorporating ligament deflection capabilities and load cells to measure knee joint compressive forces but pre-loading the ligament cables is not possible with the current device. These are some of the issues addressed in the design of the "new legform" presented below.

\section{NEW LEGFORM BONE CORE}

Bone is a unique material in that it is stiff, and yet can withstand very high strains before failure occurs. The reason bone exhibits this response is due to the fact that it is essentially a biphasic composite material composed of collagen fibers and minerals [14]. Collagen is comprised of "long fibrous material" which gives bone its strength and stiffness. The collagen is connected by a matrix of crystalline hydroxyapatite, which gives bone its hardness and overall strength [19]. The collagen and matrix layers form thin layers called lamellae [15]. The resulting cortical bone is a composite material with ultimate strains of $2-3 \%$ and a modulus of $10-15 \mathrm{GPa}$ [13]. Since bone is a composite and a complex metal structure would be needed to match both the strength and stiffness of whole bone tests, it was decided that the bone core should be made of a fiber composite material. In order for the new legform to measure bone strain in multiple loading directions (i.e. lateral-medial, anteriorposterior or some angle in between), it was decided to design cylindrical bone cores that were symmetric in the transverse plane. This will allow them to be instrumented with strain gauges in the frontal and sagittal planes so out of plane strain can be accurately computed.

\section{Design of Composite Materials}

The design and analysis of composites begins with analyzing each lamina layer of which the composite material or laminate is composed. Figure 4 shows a lamina with the fibers (principal directions) arranged at an angle theta to the coordinate directions. Lamina analysis consists of a plane stress assumption $\left(\sigma_{3}=\tau_{13}\right.$ $=\tau_{23}=0$ ), which is valid because a lamina with fibers oriented as shown would not be a practical material for applied stress in the 3- or Z-axis direction [20]. Using a plane stress state definition, the stresses in terms of strains are defined using four independent material properties: $E_{1}, E_{2}, v_{12}$, and $G_{12}$. Because the lamina has different stiffnesses, $E$, in the 1 and 2 principal directions, it is a transversely isotropic material. But since the lamina is defined in a plane stress state, it is referred to as orthotropic [21].

In order to use developed equations to analyze a laminate, which is composed of all the individual lamina layers, a relationship needs to be made between the applied stresses and the resultant forces and moments, which are referred to as line loads and moments, respectively. These loads are calculated by integrating the stresses in each lamina through the total laminate thickness, which yields the applied force and moment per unit width. Laminate analysis can be performed using the standard composite ABD matrix,

$$
\left\{\begin{array}{l}
N \\
M
\end{array}\right\}=\left[\begin{array}{ll}
A & B \\
B & D
\end{array}\right]\left\{\begin{array}{l}
\varepsilon^{o} \\
\kappa
\end{array}\right\},
$$

where $A, B$, and $D$ are laminate bending and extension stiffnesses. Equation (1) gives the line loads in terms of laminate strains and curvatures, but for the design of the bone core, the line loads will be calculated and the corresponding strains need to be found. In order to do this, the ABD matrix is inverted:

$$
\left\{\begin{array}{l}
\varepsilon^{o} \\
\kappa
\end{array}\right\}=\left[\begin{array}{ll}
A & B \\
B & D
\end{array}\right]^{-1}\left\{\begin{array}{l}
N \\
M
\end{array}\right\}
$$

Jones [22] and Davis [23] offer detailed formulations of these equations. After the total laminate strains, $\varepsilon^{\circ}$, are calculated, individual lamina strains for each ply in the principal material directions can be computed using transformation equations. This is a lengthy process but can be accomplished with computer algorithms (see Davis [23]).

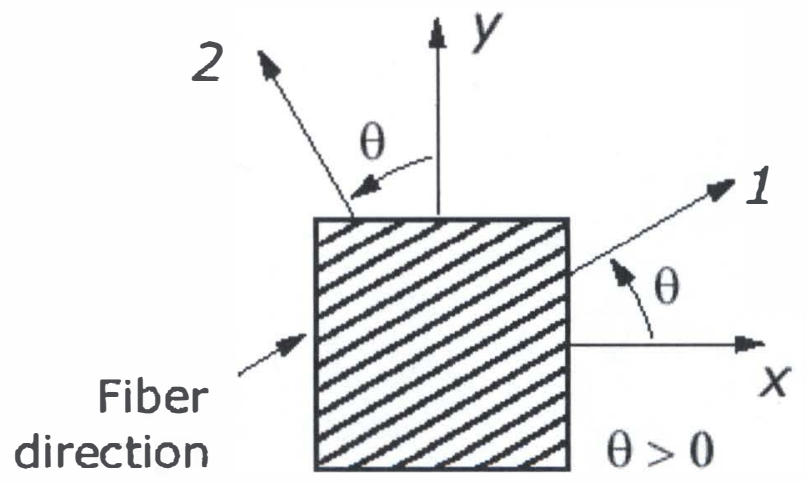

Figure 4. Lamina fiber orientation [22]

The design of the composite bone core is analyzed as a thin-walled composite tube. This is a valid assumption if the ratio of the radius to thickness of the tube is greater than 10. From the strength of materials beam equation, the stress in a beam can be found by

$$
\sigma=\frac{M c}{I}
$$

where $M$ is the applied moment, $c$ is the distance from the neutral axis, and $I$ is the moment of Inertia. In addition, the load, $P$, applied to a simply supported beam in 3-point bending can be related to the corresponding deflection, $\delta$, by 


$$
P=\frac{48 E I}{L^{3}} \delta,
$$

where $L$ is the distance between the supports. The corresponding three-point bending stiffness is

$$
k=\frac{48 E I}{L^{3}} .
$$

This equation is valid for a homogeneous, isotropic material with Young's modulus, E. For a composite beam, the preceding equation must be modified to become a valid analysis tool because of the varying stress distribution from ply to ply through the thickness of the laminate. Figure 5 shows a differential thin-walled beam element subjected to a bending moment. Line loads, $N_{x}$ and $N_{x y}$, are induced and need to be calculated in order to perform analysis on a composite member. These calculations are derived in Davis [23] with resulting line loads

$$
\begin{aligned}
& N_{x}=\frac{M c}{\pi R^{3}} \text { and } \\
& N_{x y}=\frac{V \sin \theta}{\pi R},
\end{aligned}
$$

where $V$ is the shear developed by the 3-point bending load and $\theta$ is defined in Figure 4. Once these loads are known, a composite lay-up can be designed with equation (2). This process requires much iteration to reach a specific design goal and is performed more efficiently with a computer algorithm. A Matlab script [23] was used to calculate corresponding stresses and strains given user-defined variables such as fiber and matrix material properties, number of plies, and stack angles. The maximum strain criterion was used to estimate laminate failure.

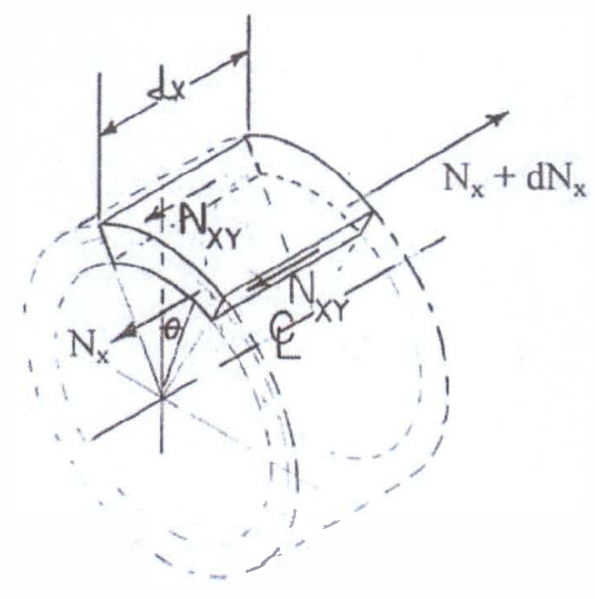

Figure 5. Differential beam element in bending [24].

\section{$\underline{\text { Tibia Design }}$}

The second slope in the lateral-medial loading direction reported by Nyquist et al. [13] was 265 (SD 70) N/mm. This is a good estimate of the 3-point bending stiffness of the tibia as the first slope was due to the fibula and soft tissue surrounding the tibia bone. Using this slope as the $k$ value in equation (5) and substituting into equation (4) yields

$$
P=265^{*} \delta
$$

based on an average support span of $250 \mathrm{~mm}$. If a 3point bending test is performed at a different span, the stiffness, $k$, must be adjusted accordingly. Using the average tibia failure moment of $315 \mathrm{Nm}$ from Nyquist et al. [13] and Kerrigan et al. [11], the maximum load, P, which the tibia bone core can support in 3-point bending is $5.12 \mathrm{kN}$, from

$$
P_{\max }=\frac{4 M_{\max }}{L} .
$$

These values were used in the composite analysis computer algorithm along with the properties of S-glass fibers and Pro-Set 117LV Resin / 226 Hardener to yield a lay-up of 8 plies at $[15,-15]_{s}$. The composite lay-up was manufactured using RTM (Resin Transfer Molding). A rubber core was used to wrap the individual S-glass lamina around and to support the lay-up during the manufacturing process. The completed bone core was allowed to cure at an elevated temperature of $400^{\circ} \mathrm{F}$ for 4 hours to increase the mechanical properties of the resin. A total of five bone cores were manufactured with variations in parameters such as lay-up technique, applied vacuum and pressure, mold prep, and cure orientation.

\section{$\underline{\text { Tibia 3-Point Test Results }}$}

The bone cores were tested in 3-point bending (see Figure 6) to failure. The support span was approximately $248 \mathrm{~mm}$. Results are shown in Figure 7 and indicate a total load capacity that is much less than anticipated by the design analysis. Post-test analysis showed failure due to excessive compressive stresses localized around the point of load location. This could be resolved by adding foam around the applicator tip in future tests. The bending stiffness was calculated to be $90-115 \mathrm{~N} / \mathrm{mm}$. This is comparable to the slope-1 stiffness reported by Nyquist but is dramatically less than what was calculated theoretically. On-going development is aimed at replacing the S-glass fibers with a Kevlar/carbon fiber mix that would increase the stiffness of the design as well as the fiber failure strains. Also, experimenting with different resins may result in a composite that compared more favorably to design values. This will be addressed in the phase 2 design of the new legform. 


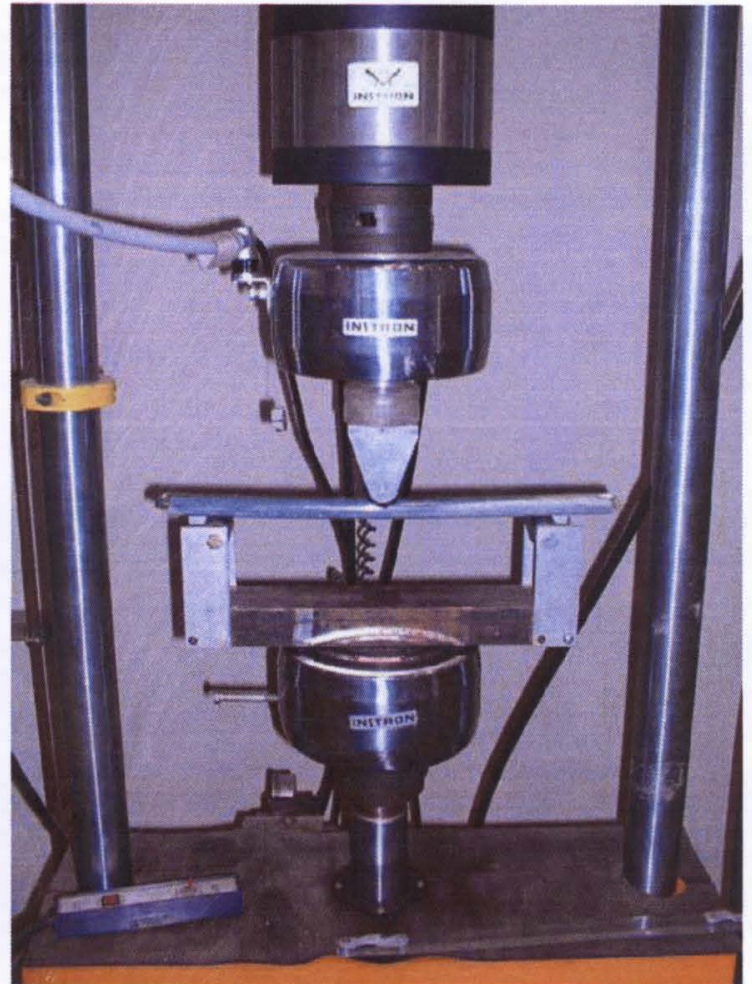

Figure 6. 3-point testing of tibia bone core using an Instron load-deflection machine.
Further iteration of the femur bone core was deferred until a more suitable composite lay-up that compared more favorably to design calculations could be found. The 3-point bending stiffness of the femur is slightly higher than the tibia at approximately $320 \mathrm{~N} / \mathrm{mm}$ from Kerrigan et al. [11]. The same design formulations and computer algorithms can be used to design a femur element in the future.

\section{NEW LEGFORM KNEE JOINT}

\section{Design and Analysis}

The design of the knee joint uses many of the features that the FLEX PLI and POLAR II have, such as ligament simulation with compression springs and cables, accurate knee geometry based on a surgeon's model, and a polyurethane meniscus. It also builds upon some existing FLEX PLI features, such as incorporating miniature potentiometers that directly measure the deflection in each ligament cable from the spring compression, not from the upper to lower knee structure. The ligament cables attach to the lower knee with adjustable nuts that allow the ligament pre-load to be precisely set with feedback from the potentiometers. Also, the load plate is piloted to the tibial plateau via two cylindrical bosses that sit directly on top of miniature compression load cells (Figure 8 ). This allows the knee

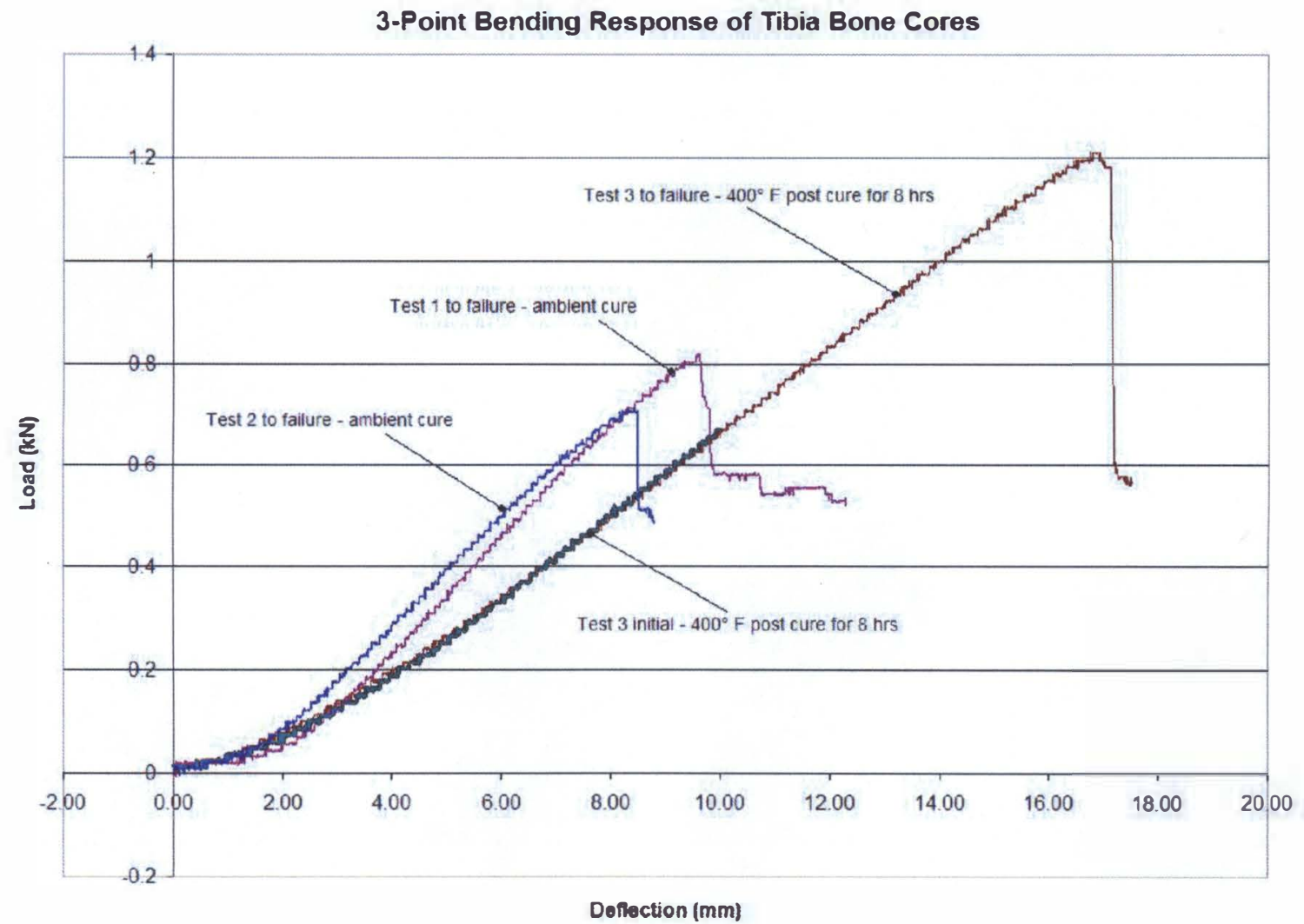

Figure 7. Composite bone core 3-point bending test results show large differences in strength properties due to variations in the manufacturing process such as composite fiber orientation and post cure temperature. 
joint compressive forces to be accurately measured including any preloading of the knee prior to testing.

As stated above, the knee joint geometry was designed from a surgeon's knee model that allowed the contact surface geometry to be designed as close to the actual human knee as possible. This allows the knee joint to be rotated or flexed so testing at angles that mimic the human gait cycle may be performed. The ligament attachment locations of the upper and lower knee were taken from the FEA knee model developed by Schuster [14]. The knee ligament stiffness values were selected based on compression spring availability and values reported in the literature (Table 1). The theoretical knee bending response was calculated from the knee ligament attachment points and spring stiffnesses with

$$
\Sigma M_{o}=r \times F
$$

This calculated bending moment depends on the point at which the moment contribution from each ligament is calculated. This was estimated as the contact point between the medal femoral condyle and the tibial plateau. While this is somewhat subjective, calculations compared favorably to the response of the FLEX PLI knee joint in cantilever type loading [25].

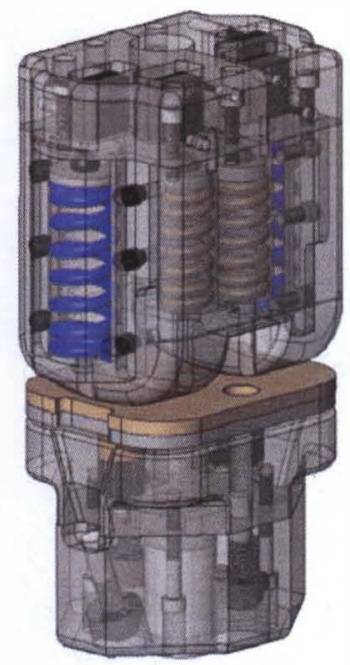

Figure 8. Solid model of knee assembly.

Table 1. Ligament stiffness values.

\begin{tabular}{|l|c|c|c|c|}
\hline Reference & $\begin{array}{c}\mathbf{A C L} \\
{[\mathbf{N} / \mathbf{m m}]}\end{array}$ & $\begin{array}{c}\mathbf{L C L} \\
{[\mathbf{N} / \mathbf{m m}]}\end{array}$ & $\begin{array}{c}\mathbf{M C L} \\
{[\mathbf{N} / \mathbf{m m}]}\end{array}$ & $\begin{array}{c}\mathbf{P C L} \\
{[\mathbf{N} / \mathbf{m m}]}\end{array}$ \\
\hline Schuster [14] & 180 & 60 & 90 & 190 \\
\hline FLEX PLI [17] & 164 & 76 & 76 & 164 \\
\hline Bartel et al. [20] & $180-242$ & - & - & 145 \\
\hline New Legform & 182 & 85 & 85 & 182 \\
\hline
\end{tabular}

The structure of the knee assembly was CNC machined from 7075-T6 billet aluminum to minimize weight and have robust properties in impact testing. The complete knee assembly is shown in Figure 9.

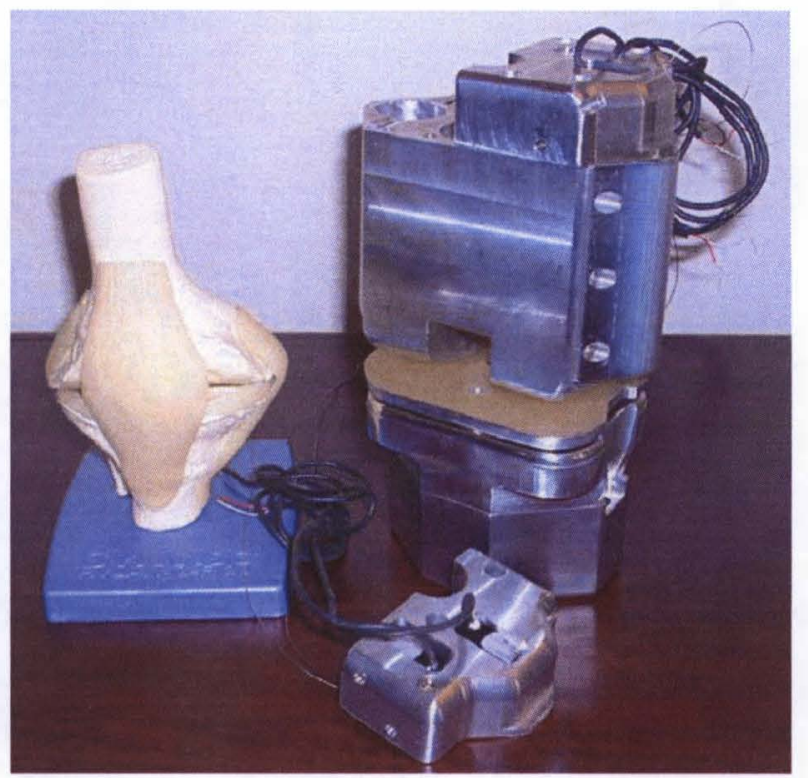

Figure 9. Knee assembly and surgeon's knee model.

\section{4-Point Testing Results of Knee Joint}

The knee joint assembly (with the composite bone cores) was tested in 4-point bending (Figure 10) to approximately 10 degrees using the Instron loaddeflection machine. Because of the test set-up, that was the maximum angle that could be achieved by this bending test. Results (Figure 11) show that the knee stiffness is within the range of PMHS tests but less than predicted. The test set-up may have contributed to the difference between analysis and test results. The lower supports rest on the composite bone cores that have a stiffness of their own. This effectively puts the composite stiffness in parallel with the knee stiffness

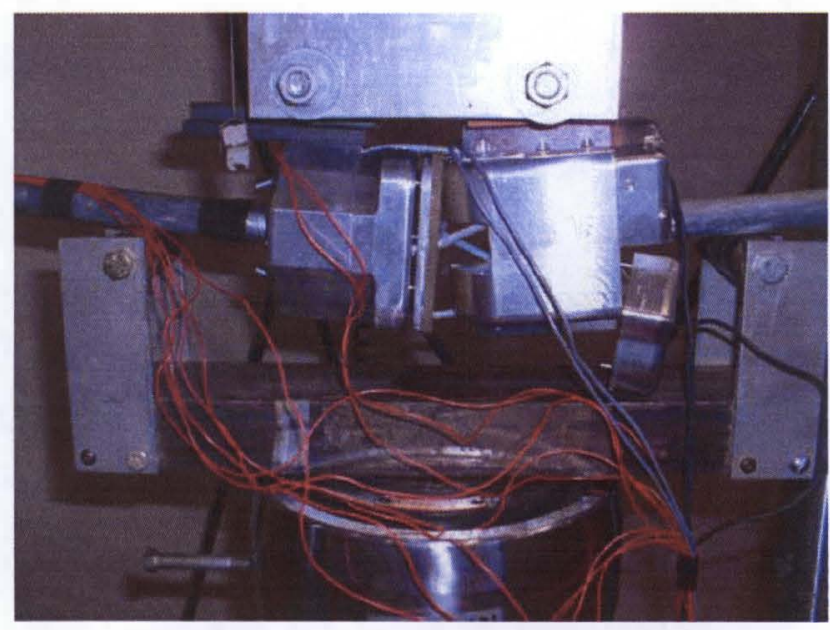

Figure 10. 4-point bending test of knee assembly. 


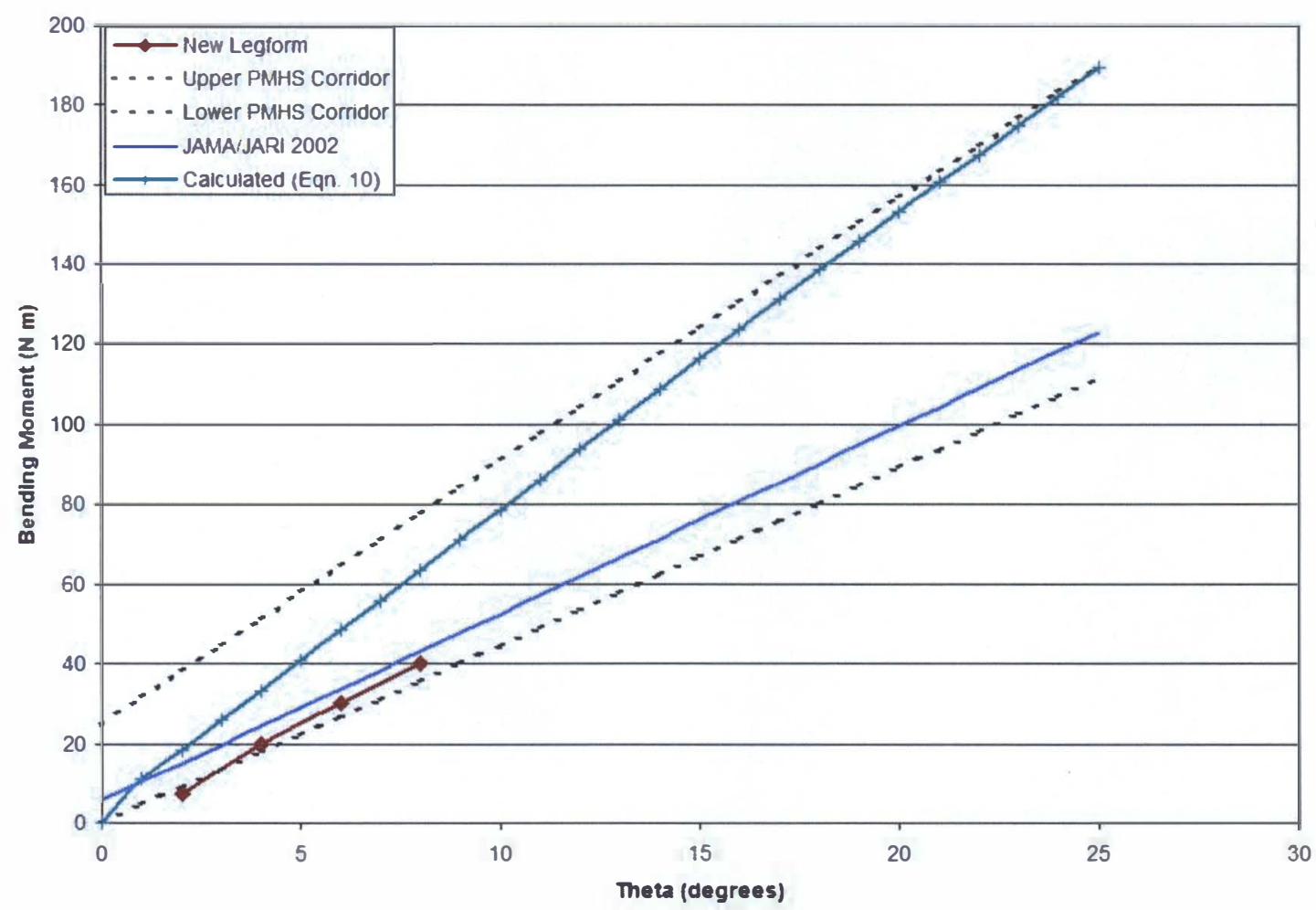

Figure 11. Cantilever type bending test comparison of New Legform with design analysis and Konosu [25] data.

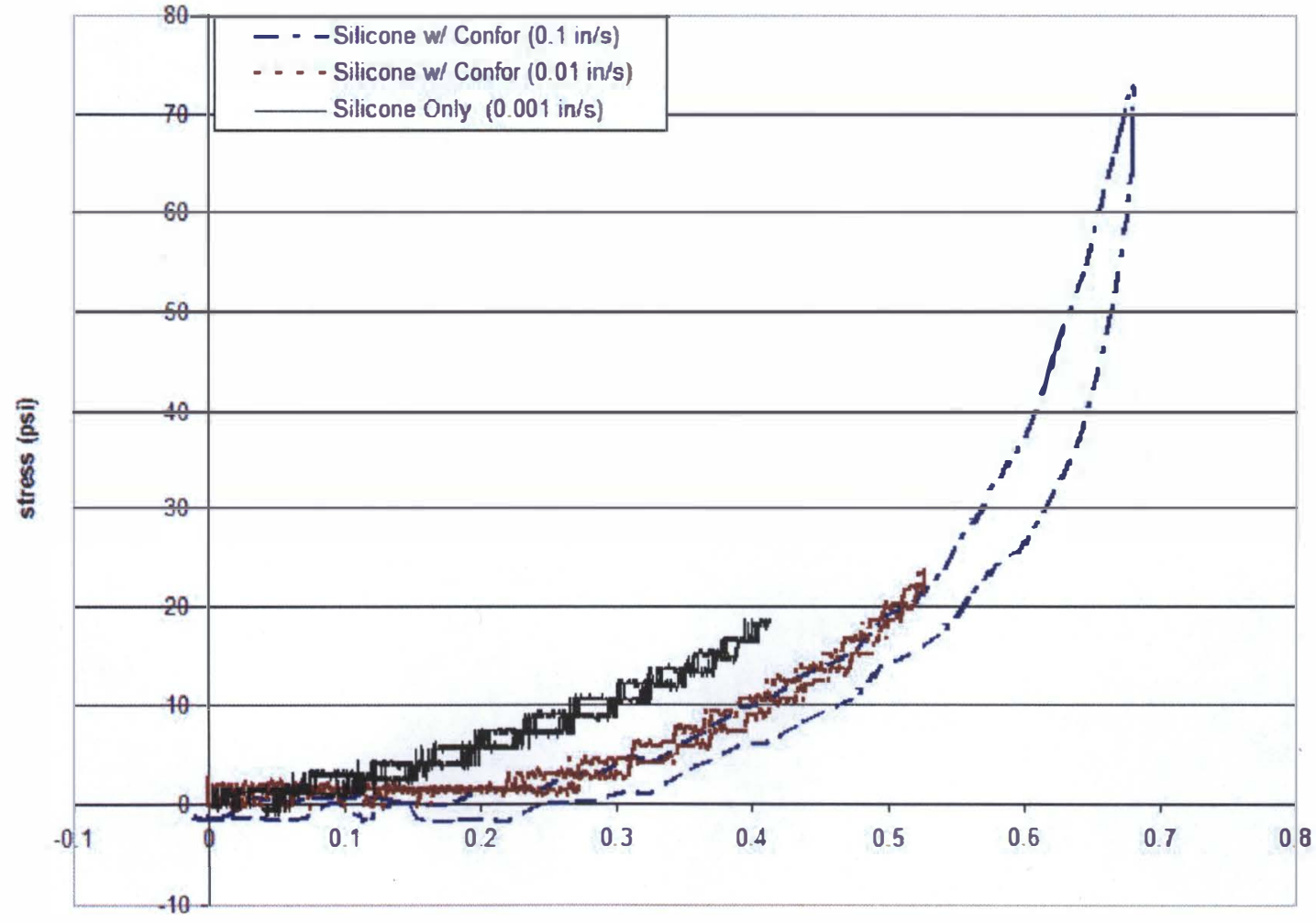

strain

Figure 12. Stress-strain curves for Silicones Inc. GI-1000 specimen with and without Confor foam at different loading rates. 
lowers the measured value. In addition, the results plotted in Figure 11 were derived from the Instron linear deflection output. This may have introduced error in the actual angular position of the knee assembly. Finally, the initial stiffness is affected by the absence of a pre-load in the knee ligament springs. Pre-loads can be adjusted to tune the bending response curve as desired in future tests.

Final calibration of the knee joint linear potentiometers and load cells will be completed in phase 2 . In addition, the knee joint bending test set-up will be modified to eliminate the effect of bone bending and increase the maximum test bend angle.

\section{NEW LEGFORM SOFT TISSUE}

The surrounding soft tissue of the new legform is designed to represent, during impact, the energy absorbing and mass properties of a $50^{\text {th }}$ percentile male. The TRL rigid, FLEX PLI, and POLAR II all include a thin layer of energy absorbing Confor foam. The new legform adds silicone rubber between the bone cores and foam covering to simulate muscle and fat tissues around the upper and lower bone elements. The silicone enables the new leg-form to achieve the correct ratios of soft-to-hard tissue in both mass and geometry. In addition, the silicone should provide similar energyabsorption capability as human muscle and fat. Unfortunately, insufficient data was available at present to confirm this assumption.

Silicones Inc. GI-1000 silicone rubber with a surface layer of Confor CF-45 foam was used to simulate muscle, fat, and skin. Stress-strain curves are shown in Figure 12 for different loading rates using silicone in series with Confor foam. The volume of silicone used for each limb was determined by the required mass properties from Robbins anthropometric data. The femur and tibia flesh diameters are $140 \mathrm{~mm}$ and $102 \mathrm{~mm}$, respectively. The thigh mass is $8.6 \mathrm{~kg}$ and the lower leg mass is $4.55 \mathrm{~kg}$, which yields a total impactor mass of $13.15 \mathrm{~kg}$.

\section{LEGFORM ASSEMBLY OVERVIEW}

The complete phase 1 legform consists of two bone cores, a knee joint assembly, silicone rubber muscles, and confor foam, all wrapped in a woven nylon 'skin.' The knee assembly has a machined aluminum frame that supports four knee 'ligament' cables, springs, and extensometers; two accelerometers; and two load cells under a tibial plateau and meniscus. The joint stiffness can be tuned by adjusting the pre-load in each ligament, allowing a range of knee joint simulations from healthy to deficient. The major components are pictured in Figure 13, with the complete assembly in Figure 14.

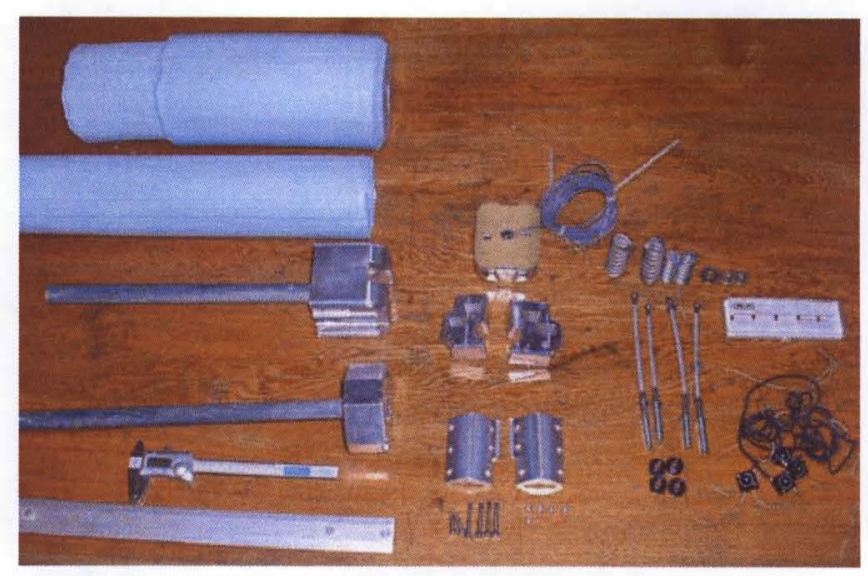

Figure 13. Overview of new legform components.

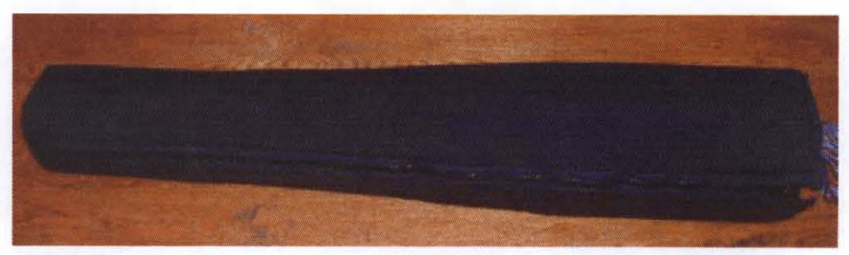

Figure 14. New legform complete with cover.

\section{CONCLUSION}

This research involved phase 1 development of a new biofidelic legform. While much progress has been made toward a pedestrian test device that more accurately simulates a human lower limb, more development is needed. The next phase will include revising the new legform so it meets design criteria without deviation and modifying the injury criteria of the EEVC to predict pedestrian injuries more accurately. Examples include using the measured ligament strains and knee compressive forces to predict knee injuries and measured strain from bone core mounted strain gauges to predict bone fractures. Phase 2 of the development to be carried out in the future includes:

1. Redesign of bone cores to theoretical design parameters of $265 \mathrm{~N} / \mathrm{mm}$ of tibia bending stiffness and $310 \mathrm{Nm}$ of maximum bending moment.

2. Calibration and correlation of legform sensors (strain gauges, load cells, potentiometers, and accelerometes) to PMHS injury tolerances.

3. Modification of EEVC injury specifications to new method of measuring pedestrian injury.

4. More detailed component level testing involving quasistatic and dynamic load cases.

5. Assembly level testing of legform.

\section{REFERENCES}

1. "Traffic Safety Facts 2002," US Department of Transportation, 2002.

2. Lawrence, G., Hardy, B., "A Study on the Feasibility of Measures Relating to the Protection of Pedestrians and Other Vulnerable Road Users Final Report," TRL Limited, June, 2004. 
3. Mizuno, Y., "Summary of IHRA Pedestrian Safety WG Activities (2003) - Proposed Test Methods to Evaluate Pedestrian Protection Afforded by Passenger Cars," JASIC, Japan, Paper \# 580.

4. "Frequencies of Pelvis/Femur Fractures for Pedestrians More Than 11 Years Old", INRETS Lab.

5. "European Experimental Vehicles Committee: Working Group 7 on Pedestrian Injury Accidents," Pedestrian Injury Accidents Proceedings of the Ninth ESV Conference, Kyoto, November, 1982.

6. "Member States Put Industrial Convenience Over Public Safety," Press Notice: European Transport Safety Council, www.etsc.be, November, 2001.

7. Bhalla, K., Bose, D., Madeley, N., Kerrigan, J., Crandall, J., Longhitano, D., and Takahashi, Y., "Evaluation of the Response of Mechanical Pedestrian Knee Joint Impactors in Bending and Loading," ESV 2003, Paper \# 429.

8. "Improved Test Methods to Evaluate Pedestrian Protection Afforded by Passenger Cars" (December 1998 with September 2002 Updates), EEVC Working Group 17 Report, www.eevc.org

9. Kajzer, J., Schroeder, G., Ishikawa, H., Matsui, Y., and Bosch, U., "Shearing and Bending Effects at the Knee Joint at High Speed Lateral Loading," SAE 1997, Paper \# 973326.

10. Takahashi, Y., Kikuchi, Y., Okamoto, M., Akiyama, A., Ivarsson, J., Bose, D., Subit, D., Shin, J., and Crandall, J., "Biofidelity Evaluation for the Knee and Leg of the Polar Pedestrian Dummy," ESV 2005, Paper \# 05-0280.

11. Kerrigan, J., Bhalla, K., Madeley, N., Funk, J., Bose, D., and Crandall, J., "Experiments for Establishing Pedestrian-Impact Lower Limb Injury Criteria," SAE 2003, Paper \# 2003-01-0895.

12. Bose, D., Bhalla, K., Rooij, L., Millington, S., Studley, A., and Crandall, J., "Response of the Knee Joint to the Pedestrian Impact Loading Environment," SAE 2004, Paper \# 2004-01-1608.

13. Nyquist, G., Cheng, R., El-Bohy, A., and King, A., "Tibia Bending: Strength and Response," SAE 1985, Paper \# 851728.
14. Schuster, P., Evaluation of the Real-World Injury Reduction Potential of the Proposed European Pedestrian 'Leg-form' Impact Test Using a Detailed Finite Element Model of the Lower Limb, Doctoral Dissertation, Michigan Technological Univ., 2000.

15. Konosu, A., Ishikawa, H., and Tanahashi, M., "Reconsideration of Injury Criteria for Pedestrian Subsystem Legform Test - Problems with the Rigid Legform Impactor," ESV 2001, Paper \# 01-S8-O263.

16. Akiyama, A., Okamoto, M., and Rangarajan, N. "Development and Application of the New Pedestrian Dummy," ESV 2001, Paper \# 463.

17. Konosu, A., and Tanahasi, M., "Development of a Biofidelic Flexible Pedestrian Legform Impactor," STAPP 2003, Paper \# 2003-22-0020.

18. Konosu, A., and Tanahasi, M., "Development of an FE Flexible Pedestrian Leg-Form Impactor (Flex-PLI 2003R) Model and Evaluation of its Biofidelity," SAE 2004 Paper \# 2004-01-1609.

19. Ramet, M., Bouquet, R., Bermond, F., and Yves, C., "Shearing and Bending Human Knee Joint Tests in Quasi-static Lateral Load," IRCOBI, 1995.

20. Bartel, Davy, and Keaveny, Mechanics and Design in Musculoskeletal Systems, El Corral Publications, 2004.

21. Nordin, M., Frankel, V.H., Basic Biomechanics of the Musculoskeletal System, Philadelphia: Lippincott Williams \& Wilkins, 2001.

22. Jones R., Mechanics of Composite Materials, $2^{\text {nd }}$ edition, New York: Brunner-Routledge, 1999.

23. Davis, J., Development of a Biofidelic Legform Impactor, Master's Thesis. California Polytechnic State University, San Luis Obispo, 2007.

24. Swanson, S.R., Introduction to Design and Analysis with Advanced Composite Materials, pp. 68-69, New Jersey, Prentice Hall, 1997.

25. Konosu, A., Tanahashi, M., "Development of a Biofidelic Pedestrian Legform Impactor Introduction of JAMA-JARI Legform Impactor Ver. 2002," ESV 2003, Paper \# 378. 